\title{
Evaluating Cancer Treatment Alternatives using Fuzzy PROMETHEE Method
}

\author{
Dilber Uzun Ozsahin $^{\text {a,b }}$, Berna Uzun ${ }^{\mathrm{a}}$, Musa Sani Musa ${ }^{\mathrm{a}}$, Abdulkader Helwan ${ }^{\mathrm{a}}$, Chidi Nwekwo Wilson ${ }^{\mathrm{a}}$, Fatih Veysel \\ Nurçin $^{\mathrm{a}}$, Niyazi Şentürk ${ }^{\mathrm{a}}$, Ilker Ozsahin ${ }^{\mathrm{a}}$ \\ ${ }^{a}$ Near East University, Near East Boulevard, ZIP: 99138Nicosia / TRNCMersin 10 - Turkey \\ ${ }^{\mathrm{b}}$ Gordon Center for Medical Imaging, Massachusetts General Hospital \& Harvard Medical School, 125 Nashua St., Boston, MA \\ 02114 U.S.A.
}

\begin{abstract}
The aim of this study is to apply the principle of multi-criteria decision making theories on various types of cancer treatment techniques. Cancer is an abnormal cell that divides in an uncontrolled manner, it is a growth (tumor) that starts when alterations in genes make one cell to grow and multiply rapidly. Eventually, these cells may metastasize to other tissues. The primary factors that influence the comprehensive treatment plan of cancer include, but not limited to genetic factors, patient general health condition, explicit characteristic of cancer, and even purpose of the treatment. Other factors which are also essential include treatment duration, cost of treatment, comfortability, side effects and percentage of survival rate. The latter factors play an important role in the course of treatment and are therefore needed in order to evaluate the several treatment procedures. The outcome of the decision-making theories on these treatment procedures will help the concerned parties such as the patients, oncologists, and the hospital management. The most common cancer treatment techniques were evaluated and compared based on certain criteria using Fuzzy PROMETHEE decision-making theory.
\end{abstract}

Keywords-Cancer treatment alternatives; multi criteria decision making; Preference Ranking Organization Method for Enrichment Evaluations (PROMETHEE); fuzzy PROMETHEE

\section{INTRODUCTION}

Cancer is an abnormal cell that divides in an uncontrolled manner, it starts when alterations in genes make one cell to grow and multiply rapidly. Eventually, these cells may metastasize to other tissues. The primary factors that influence the comprehensive treatment plan of cancer include, but not limited to genetic factors, patient general health condition, explicit characteristic of cancer, and even purpose of the treatment. There are various treatment techniques such as; chemotherapy, radiotherapy, hadron therapy, surgery, immunotherapy, and hormone therapy [1]. The primary factors that influence the treatment decision of particular cancer include; patient characteristic, disease characteristic and treatment basis [2]. Apart from the primary factors, other factors which include treatment duration, cost of treatment, Comfortability, side effects and percentage of survival rate play important role in the course of treatment and are therefore needed in order to evaluate the several treatment procedures.

PROMETHEE and Fuzzy PROMETHEE are multi-criteria decision-making techniques which are explained in Section 3. In this paper, we used Fuzzy PROMETHEE technique to evaluate the cancer treatment alternatives corresponding to their parameters.

This paper is organized as follows: In Section 1, the basic information about the cancer treatment alternatives is presented. In Section 2, PROMETHEE and fuzzy PROMETHEE techniques are shown and the proposed approach is presented. In Section 3, the results are shown. Lastly, we conclude the paper in Section 4.

\section{A. Chemotherapy}

Cancer chemotherapy uses anticancer drugs that are meant to destroy the cancer cells [3]. These drugs are being delivered orally or intravenously (injected into a vein), making its way to the bloodstream to get to the cancer cells at different parts of the body [4]. Due to the mode of operation of this therapy technique, it has unique systemic effects [5]. The way the therapy works is by slowing or inhibiting the growth of cancer cells which grow and divide rapidly. The downside of this procedure is that healthy cells that are rapidly dividing are harmed too. The noticeable side effect of this procedure arises from damage to healthy cells, but the effects may wear off when therapy is over [4].

Chemotherapy is also used alongside other modalities for treatment to ensure greater effectiveness and specificity on cancer cells. The Adjuvant chemotherapy is undergone after radiation therapy or surgery to completely destroy cancer cells that might have been left after previous treatment [5]. Neoadjuvant chemotherapy is when treatment is given to shrink the cancer cells before surgery or radiation therapy to maximize treatment efficacy [3]. Other strategies include Consolidation (given to increase cure rate), Induction (given to induce complete response to treatment), Intensification (given to effect a longer remission), Maintenance (given to delay regrowth of residual cancer cells), Palliative (given to control symptoms), and Salvage (given to patient whose symptoms have recurred or failed) [5]. The treatment regime to be used depends on the type of cancer and its severity. The above-mentioned strategies can cure, control and ease cancer symptoms [4].

\section{B. Radiotherapy}

Radiotherapy is highly competent in the treatment of a local tumor. However, clinical problems arise as a result of the radioresistance of intrinsic tumour cells to treatment. This treatment utilizes X-rays of relatively high-energy to destroy tumour cells or inhibit their growth [6]. Two main types of 
such treatment are available, External beam therapy which involves bombarding the tumour with radiation from equipment outside the body and Internal radiation treatment which uses a radioactive substance enclosed in a delivery device such as catheters, seeds, needles or wires placed near or directly into cancer [7]. Furthermore, the stage and type of cancer to be treated determine which therapy procedures should be administered. External beam treatment is used in the treatment of breast cancer whereas internal beam treatment with a radionuclide (Strontium-89) is employed to lessen bone pain resulting from breast cancer that has metastasized to the bones around the breast. Strontium-89 is administered intravenously and travels to the bone surface. The released radiation destroys tumour cells in the bones [8].

Brachytherapy is an internal radiation therapy that conveys radiation to diseased tissue from a radioactive source positioned inside the body. Brachytherapy can convey greater doses of radiation to cancer cells than external-beam radiation therapy while inflicting less damage to healthy tissue. This therapy employs the use of several techniques to treat cancer, and they are briefly discussed. Interstitial brachytherapy makes use of radiation source placed within a tumour. Intracavitary brachytherapy uses a source positioned inside a body cavity or a surgical cavity, e.g the chest cavity neighbouring a tumour [9]. Episcleral brachytherapy is used in the treatment of melanoma in the eye, with its source attached to the eye. The radioactive isotopes used in brachytherapy are enclosed in tiny pellets or "seeds". Catheters, needles, or other types of carrier are used as the delivery tools to place the seeds into patients. The resulting decay of the isotopes naturally, then gives off radiation that kills nearby cancer cells [10]. When at the location after a few weeks or months, the isotopes decay entirely and ceases to emit radiation. These seeds cause no harm when left in the body.

\section{Hadron Therapy}

This is a form of radiotherapy which uses (protons, neutrons and other ions) to treat cancer. Due to their unique radiobiological properties, these particles have the ability to penetrate tissues and deposit their peak energy. Hadrons are subatomic particles that are influenced by a strong nuclear force which binds particles in harmony within the atomic nucleus [11]. Common examples of hadrons are the neutrons and protons and that form the atomic nuclei. Protons, neutrons, and light ions such as carbon, helium, neon and oxygen are the hadrons recently used in radiotherapy [12]. These beams posses physical and radiobiological attributes which are entirely different from the X-ray and gamma ray beams used in typical radiotherapy. The interaction between charged hadrons and matter is so free which results in a welldefined spreading of the dose in depth. Light ions deposit at the end of their track a significant portion of their energy, resulting in strong local ionization that is considered largely effective against radiation-resistant tumours [13].

\section{Immunotherapy}

This is a biological process that employs certain therapeutic agents to stimulate or restore immune system functions, thereby assisting the body in fighting diseases. This therapy technique is frequently regarded as the "fourth modality" in cancer care and constitutes three forms of treatment: Cancer vaccines \& other active immunotherapy vaccine e.g. human papillomavirus vaccine, monoclonal antibodies (e.g., trastuzumab), and non-specific adjuvant therapy and immunotherapy e.g., interferon or sargramostim [14]. The effectiveness of immunotherapy could be increased by individualized therapy with suitable antibody cocktails. Immunotherapy could be regarded as an alternative treatment procedure since it can get rid off of residual tumor cells regardless of their proliferative state [15]. The aim of immunotherapy for cancer is to surmount these barriers to result in an efficient anticancer immune response.

\section{E. Hormone Therapy}

Hormone therapy also referred to as hormonal therapy or endocrine therapy, which lessens or stops the growth of tumours that have hormone receptors or are hormone sensitive by disrupting the body's ability to produce hormones or by meddling with the response of the cancer cells to the effects of hormonal changes. Hormone treatment is ineffective or not responsive when tumours are hormone insensitive and do not have hormone receptors. This therapy technique works by inhibiting the action of hormones or replacing them and also stopping the growth of cancer cells [16]. The glands produce hormones which are then circulated via the bloodstream. Furthermore, certain cancers grow as a result of the presence of some hormones. When tests indicate that the tumors receptors, then treatments that involve the use of drugs, having radiation therapy or surgery are used to lessen the release of such hormones or stop them from being potent. Estrogen, which causes the growth of some breast cancers, is produced chiefly by the ovaries. Ovarian ablation is used to stop the ovaries from producing estrogen [1].

Hormone therapy with tamoxifen is usually administered to patients with early stage cancer that can be surgically excised and cancer that spread to other parts of the body (metastatic breast cancer). Hormonal treatment using the action of estrogens or tamoxifen on cells all over the body may result in the development of endometrial cancer. Female patients on tamoxifen are required to undergo pelvic exams annually to check for any signs of cancer. However, apart from menstrual bleeding, any other vaginal bleeding should be mentioned as soon as possible to the doctor. A luteinizing hormone-releasing hormone (LHRH) agonist is administered to premenopausal patients who are diagnosed to have a hormone receptor-positive breast cancer. LHRH agonists lessen the body's progesterone and estrogen. An aromatase inhibitor is administered to patients at the premenopausal stage with hormone receptor-positive breast cancer. These inhibitors (aromatase) reduce the body's estrogen by stopping the action of the enzyme called aromatase from converting androgen into estrogen. The kinds of aromatase inhibitors are anastrozole, letrozole, and exemestane [17], [18]. For the treatment of early localized breast cancer, surgery is used to remove cancer cell using adjuvant treatments of certain aromatase inhibitors instead of tamoxifen or after 2 to 3 years of tamoxifen use. The aromatase inhibitor is also in clinical trials to compare their use to hormone therapy with tamoxifen for metastatic breast cancer treatment. Anti-estrogen treatment such as fulvestrant or megestrol 
acetate is other kinds of hormone therapy [1].

\section{F. Surgery}

Surgery is aimed at removing as much of the tumor as possible. Different types exist but the one to be performed on a patient depends on his/her choice or as recommended by the physician, based on the medical history and cancer type. Surgery is done for several reasons such as to remove more of the cancer (breast-conserving surgery (BSC) or mastectomy), investigate the spread of cancer to the axillary lymph nodes, reconstruct the breast to restore its shape, and also to relieve symptoms of advanced cancer. BCS involves removing only part of the breast with cancer also called lumpectomy, quadrantectomy, partial mastectomy or segmental mastectomy. Mastectomy is aimed at removing the entire breast and sometimes including nearby tissues. Patients have the option to choose between BCS or mastectomy, advantages of BCS is, more of the breast tissue is reserved but in most cases, there is a need for additional radiotherapy. On the other hand, most mastectomies are less likely to include a further radiotherapy. Surgery is also done to remove nearby lymph nodes. This is done in order to investigate whether the cancer has spread to the lymph node. This is very important because it helps to figure out the stage of the cancer. After most surgeries, some patients consider having their breast rebuilt to restore its appearance (breast reconstruction). Even though surgery is not likely to cure breast cancer that has reach other parts of the body, it can still be helpful in slowing the spread of the cancer or to prevent or relieve symptoms of it [19].

\section{MATERIAL AND METHODOLOGY}

\section{A. PROMETHEE (Preference Ranking Organization Method for Enrichment Evaluations)}

The PROMETHEE technique is a multi-criteria decision making technique developed by [20], [21] which is based on mutual comparison of each alternative pair with regards to each selected criteria. This model is one of the easiest and most efficient methods in conception and application compared to other MCDM methods.

The advantages of PROMETHEE technique [22]:

PROMETHEE is a user friendly outranking method.

It has been successfully implemented to the real life planning problems.

$>$ PROMETHEE I and PROMETHEE II give partial and total ranking of the alternatives respectively, while still satisfying simplicity.

The PROMETHEE method requires only two types of information: the information on the weights of the criteria considered and the decision-maker's preference function when comparing the contribution of the alternatives in terms of each separate criterion [23].

In PROMETHEE method, different preference functions are available in order to define different criteria. The preference function $\left(P_{j}\right)$ denotes the difference between the evaluations obtained with two alternatives (a and $a_{t^{\prime}}$ ) with regards to particular criterion, within a preference degree ranging from 0 to 1 . There are six different types of preference functions that can be used to implement PROMETHEE method; usual function, U-shape function, Vshape function, level function, linear function and Gaussian function.

The basic steps of the PROMETHEE method [20], [24] are:

Step 1. For each criterion j, determine a specific preference function $p_{j}(d)$.

Step 2. Define the weights of each criterion $w_{T}=$ $\left(w_{1}, w_{2}, \ldots, w_{k}\right)$. At the discretion of the decision maker, each weights of criterion can be taken equally if only their importance is equal. And also normalization can be used for the weights; $\sum_{i=1}^{k} w_{k}=1$.

Step 3. For all the alternatives, $a_{t}, a_{t^{\prime}} \in \mathrm{A}$ define the outranking relation $\pi$ :

$$
\pi\left(a_{t}, a_{t^{\prime}}\right)=\sum_{k=1}^{K} w_{k} \cdot\left[p_{k}\left(f_{k}\left(a_{t}\right)-f_{k}\left(a_{t^{\prime}}\right)\right)\right],
$$

Here $\pi(a, b)$ denotes the preference index which is a measure for the intensity of preference of the decision maker for an alternative $a_{t}$ in comparison with an alternative $a_{t^{\prime}}$ while considering all criterion simultaneously.

Step 4. Determine the leaving and entering outranking flows as follows:

Leaving (or positive) flow for the alternative $a_{t}$ :

$$
\Phi^{+}\left(a_{t}\right)=\frac{1}{n-1} \sum_{\substack{t^{\prime}=1 \\ t^{\prime} \neq t}}^{n} \pi\left(a_{t}, a_{t^{\prime}}\right)
$$

Entering (or negative) flow for the alternative $a_{t}$ :

$$
\Phi^{-}\left(a_{t}\right)=\frac{1}{n-1} \sum_{\substack{t^{\prime}=1 \\ t^{\prime} \neq t}}^{n} \pi\left(a_{t^{\prime}}, a_{t}\right)
$$

Where, $\mathrm{n}$ is the number of alternatives. Here, each alternative compared with (n-1) number of other alternatives. The leaving flow $\Phi^{+}\left(a_{t}\right)$ expresses the strength of alternative $a_{t} \in A$, while the entering flow $\Phi^{-}\left(a_{t}\right)$ denotes the weakness of alternatives, $a_{t} \in A$.

Via these outranking flows, the PROMETHEE I method can provide a partial pre-order of the alternatives and PROMETHEE II method can provide the complete pre-order based on net flow, however it doesn't give much information about the preference relations.

Step 5. Determine the partial pre-order on the alternatives of A according to following principle:

In PROMETHEE I alternative $a_{t}$ is preferred to alternative $a_{t^{\prime}}\left(a_{t} P a_{t^{\prime}}\right)$ if it satisfies the one of the following conditions:

$$
\left(a_{t} P a_{t^{\prime}}\right) \text { if ; }
$$




$$
\left\{\begin{array}{c}
\Phi^{+}\left(a_{t}\right)>\Phi^{+}\left(a_{t^{\prime}}\right) \text { and } \Phi^{-}\left(a_{t}\right)<\Phi^{-}\left(a_{t^{\prime}}\right) \\
\Phi^{+}\left(a_{t}\right)>\Phi^{+}\left(a_{t^{\prime}}\right) \text { and } \Phi^{-}\left(a_{t}\right)=\Phi^{-}\left(a_{t^{\prime}}\right) \\
\Phi^{+}\left(a_{t}\right)=\Phi^{+}\left(a_{t^{\prime}}\right) \text { and } \Phi^{-}\left(a_{t}\right)<\Phi^{-}\left(a_{t}, a_{t^{\prime}}\right)
\end{array}\right.
$$

When two alternatives $a_{t}$ and $a_{t^{\prime}}$ have the same leaving and entering flows, $a_{t}$ is in different to $a_{t^{\prime}}\left(a_{t} I a_{t^{\prime}}\right)$ :

$$
\left(a_{t} I a_{t^{\prime}}\right) \text { if: } \Phi^{+}\left(a_{t}\right)=\Phi^{+}\left(a_{t^{\prime}}\right) \text { and } \Phi^{-}\left(a_{t}\right)=\Phi^{-}\left(a_{t^{\prime}}\right) \text {. }
$$

$a_{t}$ is incomparable to $a_{t^{\prime}}\left(a_{t} R a_{t^{\prime}}\right)$ if;

$$
\left\{\begin{array}{l}
\Phi^{+}\left(a_{t}\right)>\Phi^{+}\left(a_{t^{\prime}}\right) \text { and } \Phi^{-}\left(a_{t}\right)>\Phi^{-}\left(a_{t^{\prime}}\right) \\
\Phi^{+}\left(a_{t}\right)<\Phi^{+}\left(a_{t^{\prime}}\right) \text { and } \Phi^{-}\left(a_{t}\right)<\Phi^{-}\left(a_{t^{\prime}}\right)
\end{array}\right.
$$

Step 6. Determine the net outranking flow for each alternative

$$
\Phi^{n e t}\left(a_{t}\right)=\Phi^{+}\left(a_{t}\right)-\Phi^{-}\left(a_{t}\right)
$$

Via PROMETHEE II, the complete pre-order can be obtained by the net flow and defined by:

$a_{t}$ is preferred to $a_{t^{\prime}}\left(a_{t} P a_{t^{\prime}}\right)$ if $\Phi^{\text {net }}\left(a_{t}\right)>\Phi^{\text {net }}\left(a_{t^{\prime}}\right)$

a is indifferent to $a_{t^{\prime}}\left(a_{t} I a_{t^{\prime}}\right)$ if $\Phi^{\text {net }}\left(a_{t}\right)=\Phi^{\text {net }}\left(a_{t^{\prime}}\right)$.

Basically the better alternative is the one having the higher $\Phi^{\text {net }}\left(a_{t}\right)$ value.

\section{B. Fuzzy PROMETHEE (F-PROMETHEE)}

There has been few research based on the approach of fuzzy PROMETHEE (F-PROMETHEE). Among the few researchers that have applied fuzzy PROMETHEE include [25]-[29]. In real life conditions, most times it is difficult to collect crisp data to define a problem properly and make an optimal decision. Using Fuzzy sets gives the decision maker the ability to define the problem under the vague condition which is more realistic.
The main aim of the Fuzzy PROMETHEE model was proposing a comparison between two fuzzy sets. Yager [30] found an index which is determined with the center of weight of the surface of the membership function to compare the fuzzy numbers. Yager defined the magnitude of a triangular fuzzy numbers $\tilde{F}=(N, a, b)$, which is equivalent to $\tilde{F}=$ $(N-a, N, N+b)$, corresponding to center of triangle with the $Y I=(3 N-a+b) / 3$ formula. In our F- PROMETHEE application we applied Yager index to compare the fuzzy numbers.

\section{Application}

First, we defined the importance of the parameters with the linguistic scale as seen in Table 1 and then we applied Yager index to obtain the weight for each criterion as seen in Table 2 .

TABLE I. LINGUISTIC SCALE FOR IMPORTANCE

\begin{tabular}{|l|l|l|}
\hline $\begin{array}{l}\text { Linguistic scale for } \\
\text { evaluation }\end{array}$ & $\begin{array}{l}\text { Triangular fuzzy } \\
\text { scale }\end{array}$ & $\begin{array}{l}\text { Importance ratings of } \\
\text { criterions }\end{array}$ \\
\hline Very high (VH) & $(0.75,1,1)$ & $\begin{array}{l}\text { Cost of Machine, Cost of } \\
\text { Treatment, Percentage of } \\
\text { Survival }\end{array}$ \\
\hline Important (H) & $(0.50,0.75,1)$ & \\
\hline Medium (M) & $(0.25,0.50,0.75)$ & Treatment Time \\
\hline Low (L) & $(0,0.25,0.50)$ & \\
\hline Very low(VL) & $(0,0,0.25)$ & Comfortability \\
\hline
\end{tabular}

After we collect all the data for the alternatives, we applied them to Visual PROMETHEE Decision Lab program as shown in Table 2. We used the V-shape function for treatment

\begin{tabular}{|c|c|c|c|c|c|}
\hline Criteria & Treatment Time & Cost of Machine & Cost of Treatment & Comfortability & $\begin{array}{l}\text { Survival } \\
\text { Percentage }\end{array}$ \\
\hline Unit & weeks & $\$$ & $\$$ & $y / n$ & $\%$ \\
\hline \multicolumn{6}{|l|}{ Preferences } \\
\hline$(\min / \max )$ & $\min$ & $\min$ & $\min$ & $\max$ & $\max$ \\
\hline Weight & 0,50 & 0,92 & 0,92 & 0,08 & 0,92 \\
\hline Preference Fn. & V-shape & Linear & Linear & Level & Level \\
\hline \multicolumn{6}{|l|}{ Evaluations } \\
\hline Chemotherapy & 104 & 0 & 7470 & Yes & 70 \\
\hline Radiotherapy & 6 & 3000000 & 5333 & Yes & 55 \\
\hline Hadron therapy & 6 & 162500000 & 13833 & Yes & 97 \\
\hline Immunotherapy & 104 & 0 & 27925 & Yes & 50 \\
\hline Surgery & 2 & 0 & 32500 & No & 86 \\
\hline Hormone therapy & 260 & 0 & 2256 & Yes & 69 \\
\hline
\end{tabular}
time, linear function for cost of machine and cost of treatment and level preference function for comfortability and percentage of survival.

TABLE II. VISUAL PROMETHEE APPLICATION FOR THE CANCER TREATMENT ALTERNATIVES

\section{RESULTS}

Result from Table 3 shows that hadron therapy with the highest survival rate, short treatment time and noninvasiveness tops the list of alternatives and therefore, it will be more beneficial to the patient. The criteria for cost of machine were de-activated in getting the result as it does not benefit the patient.

Result from Table 4 shows that Surgery with the highest treatment cost will be more advantageous to the hospital.
The criteria for cost of treatment were de-activated while obtaining this ranking because it has no benefit on the hospital side.

Fig. 1 shows the positive and negative side of the Treatment Alternatives for each selected criteria. We used Decision Lab visual PROMETHEE program to have the result. This program is user friendly and the decision maker can change the criteria and also the weight for criterion easily and can compare the devices according to criteria they wish. 
TABLE III. COMPLETE RANKING OF CANCER TREATMENT Alternatives IN TERMS OF PATIENT

\begin{tabular}{|lcc|}
\hline $\begin{array}{l}\text { Rank of alternatives } \\
\text { Negative outranking flow }\end{array}$ & Net flow & Positive outranking flow \\
\hline 1 Hadron therapy & 0,4931 & 0,5041 \\
& & 0,0110 \\
\hline 2 Surgery & 0,3741 & 0,4501 \\
& & 0,0760 \\
\hline 3 Chemotherapy & $-0,0446$ & 0,2314 \\
& & 0,2760 \\
\hline 4 Radiotherapy & $-0,1152$ & 0,2000 \\
& & 0,3152 \\
\hline 5 Hormone therapy & $-0,2446$ & 0,1521 \\
& & 0,3967 \\
\hline 6 Immunotherapy & $-0,4628$ & 0,0413 \\
& & 0,5041 \\
\hline
\end{tabular}

TABLE IV. COMPLETE RANKING OF CANCER TREATMENT ALTERNATIVES IN TERMS OF HOSPITAL

\begin{tabular}{|lcc|}
\hline $\begin{array}{l}\text { Rank of alternatives } \\
\text { Negative outranking flow }\end{array}$ & Net flow & Positive outranking flow \\
\hline 1 Surgery & 0,5262 & 0,6022 \\
& & 0,0760 \\
\hline 2 Hadron therapy & 0,1129 & 0,5041 \\
& & 0,3912 \\
\hline 3 Chemotherapy & 0,1074 & 0,3835 \\
& & 0,2760 \\
\hline 4 Hormone therapy & $-0,0926$ & 0,3041 \\
& & 0,3967 \\
\hline 5 Immunotherapy & $-0,3107$ & 0,1934 \\
& & 0,5041 \\
\hline 6 Radiotherapy & $-0,3433$ & 0,2760 \\
& & 0,6193 \\
\hline
\end{tabular}

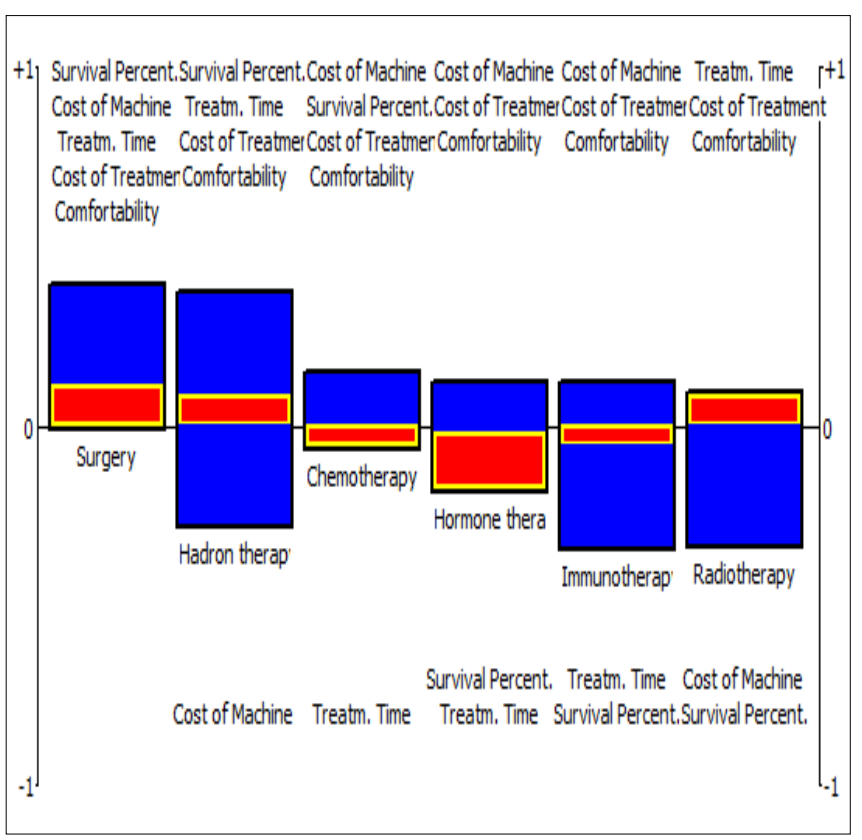

Fig. 1. PROMETHEE evaluation results.

\section{CONCLUSION}

Using fuzzy PROMETHEE as a multi-criteria analysis technique, we were able to achieve good decision results by incorporating fuzzy input data. The fuzzy PROMETHEE method was applied on various cancer treatment alternatives. This study indicates that the proposed method simply and practically provides advanced alternative solutions to decision-making problems. The results of this ranking give the decision makers or organizations the ability to choose or improve their treatment procedures. The study can be improved by adding more criteria to the alternatives.

With the Fuzzy PROMETHEE technique, the problem of decision making for the fuzzy data is solved. This technique has proven to be very efficient in many fields when compared to other decision making techniques. The outcome of this study will benefit the patient, likewise the hospital management in making relevant decision as to managing the hospital or the patient. Other works have been done to compare nuclear imaging devices and image reconstruction techniques. This method can also be extended to other aspect of medical decision making.

\section{REFERENCES}

[1] T. Beyer, D.W. Townsend, T. Brun, P.E. Kinahan, M. Charron , R. Roddy, J. Jerin, J. Young, L. Byars, R. Nutt, 'A combined PET/CT scanner for clinical oncology', The Journal of nuclear medicine, 41(8), 1369-1379, 2000.

[2] Lymphomation.org. (2017). Factors that Influence Treatment Decisions. [online] Available at: http://www.lymphomation.org/treatmentdecisions.htm [Accessed 18 Oct. 2017].

[3] R. Airley, (2009). Cancer chemotherapy. 1st ed. Chichester: WileyBlackwell, pp.55-111.

[4] K.B. Andreas, N. Stephan, Z. Sibylle, B. Ambros, J.K. Bernd H. Ken, S. Klemens, W. Hans-Juergen, T.R. Ernst, S. Markus and D. Alexander SPECT/CT, Journal of Nuclear Medicine, 49(8), 1305-1319, 2008.

[5] Baquiran, D. and Gallagher, J. (2011). Lippincott's Cancer Chemotherapy Handbook. 1st ed. Philadelphia: Wolters Kluwer Health, pp.3-7.

[6] A. Barrett, and S. Morris, (2009). Practical Radiotherapy Planning Fourth Edition. 1st ed. Taylor \& Francis.

[7] Training.seer.cancer.gov. (2017). SEER Training: Types of Radiation Therapy. [online] Available at: https://training.seer.cancer.gov/treatment/radiation/types.html [Accessed 10 April 2017].

[8] E. Silberstein, Systemic radiopharmaceutical therapy of painful osteoblastic metastases. Seminars in Radiation Oncology, 10(3), pp.2, 2000.

[9] V. DeVita, T. Lawrence, and S. Rosenberg, (2011). Cancer. 1st ed. Philadelphia: Wolters Kluwer/Lippincott Williams \& Wilkins Health.

[10] L.F. Fajardo, M. Berthrong, and R.E. Anderson, Radiation pathology. Oxford University Press, 2001.

[11] R. Orecchia, A. Zurlo, A. Loasses, M. Krengli, G. Tosi, S. Zurrida, P. Zucali, and U. Veronesi, 'Particle beam therapy (hadrontherapy): basis for interest and clinical experience', European Journal of Cancer, 34(4), pp.459-468, 1998.

[12] National Research Council. (2013). Nuclear Physics: Exploring the Heart of Matter. National Academies Press.

[13] T. Mitin, and A. Zietman, 'Promise and Pitfalls of Heavy-Particle Therapy', Journal of Clinical Oncology, 32(26), pp.2855-2863, 2014. 
[14] Y. Shih, L. Elting, A. Pavluck, A. Stewart, and M. Halpern, 'Immunotherapy in the Initial Treatment of Newly Diagnosed Cancer Patients: Utilization Trend and Cost Projections for Non-Hodgkin's Lymphoma, Metastatic Breast Cancer, and Metastatic Colorectal Cancer', Cancer Investigation, 28(1), pp.46-53, 2009.

[15] S. Braun, F. Hepp, H. Sommer, and K. Pantel, 'Tumor-antigen heterogeneity of disseminated breast cancer cells: Implications for immunotherapy of minimal residual disease', International Journal of Cancer, 84(1), pp.1-5, 1999.

[16] Hormonal Therapy for Treating Breast Cancer | Breastcancer.org", Breastcancer.org, 2017. [Online]. Available: http://www.breastcancer.org/treatment/hormonal. [Accessed: 22- Oct2017].

[17] A.U. Buzdar, and G. Hortobagyi, 'Update on endocrine therapy for breast cancer', Clinical Cancer Research, 4(3), pp.527-534, 1998.

[18] V. Bock, M. Friedlander, D. Waring, S. Kossard, and G. Wood, (2013). 'Cutaneous adverse effects of hormonal adjuvant therapy for breast cancer: A case of localized urticarial vasculitis following anastrozole therapy and a review of the literature', Australasian Journal of Dermatology, 55(4), pp.282-285, 2013.

[19] Surgery for breast cancer. 2017. Surgery for breast cancer. [ONLINE] Available at: https://www.cancer.org/cancer/breastcancer/treatment/surgery-for-breast-cancer.html. [Available $10 \mathrm{march}$ 2017].

[20] J.P. Brans, P. Vincle. 'A preference ranking organization method', Management Science, 31(6), 647-656, 1985.

[21] J.P. Brans, P. Vincke, B. Mareschal, 1986. 'How to select and how to rank projects: the PROMETHEE method', European Jornal of Operational Research, 24, 228-238, 1986.

[22] F. Ulengin, Y. Topçu, S.O. Sahin, An Integrated decision aid system for
Bosporous water crossing problem. Eur. J. Oper. Res. 134, 179- 192, 2001.

[23] C. Macharis, J. Springael, K. de Brucker, A. Verbeke, 'PROMETHEE and AHP: the design of operational synergies in multicriteria analysis. Stengthening PROMETHEE with ideas of AHP', European Journal of Operational Research, 153(2), 307-317, 2004.

[24] Geldermann, J., Spengler, T., Rentz, O.: Fuzzy outranking for environmental assessment. Case study: iron and steel making industry, , 115(1), 45-65 (2000).

[25] M., Goumas, V. Lygerou, An extension of the PROMETHEE method for decision making in fuzzy environment: ranking of alternative energy exploitation projects. European Journal of Operational Research, 123, 606-613, 2000.

[26] R.U. Bilsel, G. Buyukozkan, D. Ruan, 'A fuzzy preference ranking model for a quality evaluation of hospital web sites', International Journal of Intelligent System, 21(11), 1181-1197, 2006.

[27] W.C. Chou, W.T. Lin, C.Y. Lin, 'Application of fuzzy theory and PROMETHEE technique to evaluate suitable eco-technology method: a case study in Shismen reservoir watershed', Ecological Engineering, 31, 269-280, 2007.

[28] G. Tuzkaya, B., Gülsün, C. Kahraman, D. Özgen, 'An integrated fuzzy multi-criteria decision making methodology for material handling equipment selection problem and an application', Expert Systems, Appl. 37(4), 2853-2863, 2010.

[29] A. Ozgen, G. Tuzkaya, U.R. Tuzkaya, D. Ozgen, 'A Multi-Criteria Decision Making Approach for Machine Tool Selection Problem in a Fuzzy Environment', International Journal of Computer Intelligence System, 4(4), 431-445, 2011.

[30] J.M. Ollinger, J.A. Fessler, 'Positron-emission tomography. IEEE Signal Processing Magazine2, 14(1), 43-55, 1997. 